\title{
Extracting identity and location information from briefly presented letter arrays
}

\author{
CAROL L. KRUMHANSL and EWART A. C. THOMAS \\ Stanford University, Stanford, California 94305
}

\begin{abstract}
The effect on identification and location of briefly presented targets (letters and blanks) of number of targets, retinal location, and direction and distance of simultaneously presented targets is investigated. In three different conditions, one, two, or three targets are presented on a trial with the location of targets varying from trial to trial. A model is proposed to describe identification and location performance in the one-letter condition. This model distinguishes between two sets of parameters, perceptual parameters and decision parameters, and assumes that in terms of these parameters, identification and location are independent processes. The perceptual and decision parameters of the model, estimated by assuming that these parameters are linearly related to retinal location, indicate that retinal location has a different effect on the decision parameters for both identification and location than on the perceptual parameters. Variables affecting the performance decrements found when multiple letters are presented are evaluated in terms of models stated for the multiple letter conditions. Estimates of the effects of distance from the fixation point and interletter distance indicate that, as both of these variables increase, performance decreases. In the three-letter condition, a serial position effect is found, with performance on center letters being relatively poor. A peripherally presented letter is found to have a large detrimental effect on performance, and the effect increases as interletter distance increases. Location of correctly identified targets follows many of the same patterns as identification performance in the multiple letter conditions. In addition, there is a tendency for incorrectly located targets to be moved foveally and to move to positions occupied by other targets on the trial. The possibility of a limited-capacity attention mechanism operating in the present experiment is discussed.
\end{abstract}

Identification of letters presented in tachistoscopic displays has been found to be affected by various response variables, e.g., order of report, and various display variables, e.g., location in the visual field (see Estes, Allmeyer, \& Reder, 1976, and Wolford, 1975, for reviews of this literature). The present paper is concerned only with the effects of display variables in a report paradigm. This choice of focus relies on arguments that display variables have discernible effects even after one controls for response variables (Estes et al., 1976; Estes \& Wolford, 1971; Townsend, Taylor, \& Brown, 1971). Some of the display variables that have been investigated are (1) number of letters to be reported (targets) and number of other letters in the display (distractors) (Estes, 1972, 1974; Sperling, 1960), (2) level of confusability between targets and distractors and among distractors (Estes, 1972, 1974; Gardner, 1973), (3) the size of the interletter distance (Collins

The work was supported by a National Institutes of Health training grant (MH-10478-09) to the first author, a National Science Foundation grant (GB-43275) to the second author, and a National Institute of Mental Health grant (MH-21747) to Richard C. Atkinson. The authors are grateful for the assistance of Richard C. Atkinson, and the helpful comments of Edward E. Smith and two reviewers on an earlier version of this manuscript. Wesley Mah assisted in running subjects. Reprint requests may be sent to Carol L. Krumhansl, Department of Psychology, Stanford University, Stanford, California 94305.
\& Eriksen, 1967; Crovitz \& Schiffman, 1965; Eriksen \& Eriksen, 1974), (4) the replacement of certain letter positions in the string with blank spaces (Estes \& Wolford, 1971; Shaw, 1969; Wolford \& Hollingsworth, 1974b), (5) the relative position of a target within the array (Estes et al., 1976; Wolford \& Hollingsworth, 1974c), (6) the absolute position of a target letter in the visual field (Crovitz \& Schiffman, 1965; Estes et al., 1976; Wolford \& Hollingsworth, 1974c), and (7) the duration of the stimulus presentation (Estes et al., 1976; Taylor \& Brown, 1972; Townsend, Taylor, \& Brown, 1971). The present study will address a number of issues raised in the studies mentioned above.

First, in order to account for many of the main and interaction effects found when display variables are manipulated, Estes (1972) proposed a model in which it is assumed that retinal location, defined here as the distance from a fixation point, affects the probability of detecting a target because the density of input channels to feature detectors decreases from the center to the periphery of the visual field. Moreover, the fact that increasing the number of distractors lowers detection probability, particularly when distractors are confusable with the target, suggests that at a feature extraction stage excitation of a particular input channel inhibits other channels going to the same or nearby feature detectors. 
Since inhibition between channels is expected to increase as the distance between them decreases, performance would be expected to decline as the spacing between the presented targets decreases. The proposed distribution of input channels, taken together with inhibition between channels, predicts an interaction between the location of a target and the number of nearby targets, so that the decline in performance found with increased distance from the center of the fovea should be larger when more targets are present in the vicinity. In particular, an interaction (statistical) should be found between absolute position (retinal location) and the relative position of the letter within the string (end vs. middle letter position). In some studies, middle letters are more affected by retinal location than end letters (Estes \& Wolford, 1971; White, 1970; Wolford \& Hollingsworth, 1974c), whereas in the study of Estes et al. (1976), in which the letter strings were presented unilaterally, the largest retinal location effect was found for the letter at the foveal end of the letter string. The latter study also differed from the former studies in that, in the Estes et al. (1976) study, the arrays were embedded in a string of mask characters and the letters were presented over a wider range of visual angle. The influence of these factors on the interaction between absolute position and string position needs to be further investigated. In general, the model proposed by Estes (1972) can successfully deal with many aspects of existing data on the effect of various display variables on letter identification.

A second issue is the locus of the interference effects found between presented targets. The model proposed by Estes (1972) focuses on inhibitory effects among input channels during the feature extraction stage. A number of other studies (see, for example, Estes, 1974; Gardner, 1973; and Shiffrin \& Geisler, 1973) have suggested that interference effects between targets in a detection task may occur at the decision stage rather than, or in addition to, the perceptual stage. The general approach taken in the studies which focus on the decision stage is to assume that characters independently activate representations in memory and that adding additional (noise) characters to a display complicates the decision process, since degraded inputs of nontarget characters may be mistaken for targets and give rise to incorrect detection responses. The question remains unresolved as to which of the two approaches provides a better account of performance in tachistoscopic letter experiments.

A third issue concerns the role location information may play in the letter identification process. Estes et al. (1976) investigated the effect retinal location, serial position, and viewing time have on locating as well as identifying targets. Since the Estes (1972) model assumes that the density of input channels to feature detectors decreases from the center to the periphery of the visual field, greater uncertainty concerning target position might be expected at more peripheral locations. In support of this expectation, the Estes et al. (1976) study found that the difficulty in locating correctly identified targets increased with distance from the center of the visual field. In addition, location performance was poorer at central serial positions than at end positions, and these effects were present even at extended viewing times. Finally, it was found that transposition errors tended to occur in a central direction, and that transpositions tended to terminate in the positions of letters which were not reported at all on the trial. It is hypothesized that positional uncertainty may play a role in the interference effects found when multiple letters are presented in that, if two letters are such that their location distributions overlap, representations of both letters may be simultaneously activated and may compete for access to the response system. Since the quality of location information declines with distance from the center of the visual field, the interference effects would be expected to increase at peripheral locations. Also, since letters at the ends of letter strings in their study are flanked on one side by an unchanging mask character, performance is relatively unimpaired since the mask character can be readily rejected at the decision level. Finally, since positional uncertainty is biased toward the center of the fovea, replacing a peripheral neighbor by a blank space or unchanging mask character should have a larger facilitating effect than replacing a central neighbor by a blank or unchanging mask character. Support for this last prediction comes from the Estes et al. (1976) study as well as from other studies (see for example, Shaw, 1969; Wolford \& Hollingsworth, 1974b). In the study of Estes et al. (1976), positional uncertainty was hypothesized to have a detrimental effect on identification performance since response competition occurs when two location distributions overlap. A somewhat different approach was taken in the study of Wolford (1975), in which it was assumed that positional uncertainty, in the form of permutations in the order of the extracted features during the processing of information in the sensory store, causes a problem in the identification of characters. His model provides a good account of identification performance in a variety of report experiments. However, it is unclear how the model would extend beyond predicting identification performance (omission and confusion errors) to predicting location performance (transposition and inversion errors). In any case, the model assumes that the transition probability for a feature moving from a feature group decreases with distance from the fovea (his Equations 2 and 3), so that it is unlikely that the model would provide a good account of location performance. 
Finally, an issue of interest is whether the notion of a limited-capacity attention mechanism, rather than the notion of lateral interference, provides a better account of the data on letter identification and location. While attention models have traditionally focused on the role of attention in determining identification performance, there is no reason that it is not equally applicable to location performance. All of what follows, therefore, will apply to the processing of both types of information. According to the model proposed by Rumelhart (1970), there is a limit to the amount of perceptual or attentional capacity available for processing of items, and when the capacity is shared between items, the result is a decrement in the processing efficiency for any given item. This general model might be augmented in the following way. Suppose, for example, that owing to limitations inherent in the visual system, more attention is required at peripheral locations than at central locations for a certain level of performance, and that peripheral targets tend to be allotted a greater proportion of the available attention. Greater allocation of attention to the periphery might constitute a strategy for compensating for perceptual limitations at these locations and is similar to the ends-first processing model in studies of Coltheart (1972), Merikle and Coltheart (1972), and Merikle, Coltheart, and Lowe (1971). Breitmeyer and Ganz (1976) have further noted the correlation between the notion that attention is initially concentrated at the periphery with the relatively high concentration of transient neurons in the periphery, which are thought to direct selective attention mechanisms. The usual assumption made in attentionsharing approaches is that attention is shared across items and not across distance per se. In the situation in which (nonconfusable) noise characters are interposed between target characters when intertarget distance increases, it might be assumed that some attention is allocated to the intervening noise characters. In this case, it could be assumed that increasing intertarget distance would be associated with a decrease in performance. This prediction is generally opposite that predicted by an interference hypothesis, namely, that interference should decrease, hence performance should improve, as intertarget spacing is increased. The predictions made by these assumptions are consistent with the effects found for retinal location, presence vs. absence of a peripheral target, and serial position when the letter strings span the fovea. The assumptions, however, are not sufficient to explain why serial position curves are typically U-shaped when letter strings are confined to one side of the fixation point. They do, however, suggest that if display variables are found to have similar effects on both location and identification of target items, it may be that the dis- play variables are affecting the allocation of attention, and thus producing the covariance between the two performance measures, rather than that the quality of location information is directly influencing the level of identification performance.

The present study is concerned with the identification and location of targets (letters and blank spaces) in an array of fixed length consisting of targets and noise characters. The number and location of targets are varied, with the restriction that, on any trial, all targets are presented on the same side of the fixation point. The subject's task is to report all of the targets in their correct locations, so that the response measures of interest are which targets are reported and which locations are chosen as target locations. We shall not examine the characteristics of incorrectly identified targets, i.e., confusion errors (see, for example, Townsend, 1971), but we shall look at incorrectly chosen locations by examining where targets are located in the response protocols.

Our approach to the issue mentioned above will be twofold. First, we propose and test a model to be stated presently which assumes that in the simple situation in which only one target is presented on a trial for identification and location, the two processes are carried out independently. We will identify two sets of parameters in the model, those referring to perceptual processes and those referring to decision processes, and these parameters will be assumed to be linear functions of retinal location. The relative magnitude of the slopes of these functions will give some indication of the locus of the retinal location effect. Second, we investigate the effect in multipleletter displays of such variables as retinal location of a target, and number, distance, and direction (foveal or peripheral) of other simultaneously presented targets on both identification and location performance. The results are considered with respect to both the notion of lateral interference, as suggested by Estes (1972), and the notion of a limited-capacity attention mechanism, as outlined above.

The model for single-target performance is proposed to describe performance in the task in which a subject is asked on a trial to locate and identify a single presented target. Any given trial may result in one of five possible outcomes: no response, incorrect identification and incorrect location, correct identification but incorrect location, incorrect identification but correct location, and correct identification and correct location. The main assumption of the model is that identification and location are processes which are carried out independently. The assumption of independent processing seems to be the appropriate "null hypothesis" for testing against most approaches which have assumed that the identification process is dependent on the location process (Estes et al., 1976; Logan, 1975a; Wolford, 1975). 
Next, it is assumed that with respect to the identification and location processes, the subject is in one of three sensory states $D_{0}, D$, and $D^{*}$, corresponding to no useful information, partial or fallible information, and complete information. These three states are analogous to those of discrete-state detection models proposed by Luce (1963) and Krantz (1969). The state $D_{0}$ is regarded as the source of no response on a trial. The state $D^{*}$ is considered to be equivalent to the "primary detect" state proposed by Estes (1972). The probabilities of being in states $D_{0}, D$, and $D^{*}$ are $a_{0}, a$, and $a^{*}$, respectively, for identity information, and $b_{0}, b$, and $b^{*}$, respectively, for location information. The perceptual information is then used to make a decision as to the identity and location of the target. It is assumed that if the subject is in state $D_{0}$ with respect to identity (location) information, no decision is made as to the identity (location) of the target. In the state $D$, the probability of making a correct identification (location) decision is a $\alpha(\beta)$ and the probability of making an incorrect identification (location) decision is $1-\alpha(1-\beta)$. If the subject is in state $D^{*}$ with respect to identity (location) information, a correct identification (location) decision is made with probability 1. Finally, at the response level, it is assumed that no response is made unless the decision stage yields both identification and location decisions. This implies that no response is made if the subject is in state $D_{0}$ with respect to either identity or location information.

The distinction made in the model just proposed between perceptual and decision parameters is to some extent arbitrary. For example, in the intermediate state $\mathrm{D}$, it is impossible to distinguish between whether the subject has less perceptual information available or has full information but only chooses to process some of it. The present formulation was chosen to reflect the distinction between variables which would influence the amount or quality of information (e.g., duration) from those which would influence the decision process (e.g., level of confusability between targets presented at wide separations). However, the arbitrariness of the distinction between the perceptual and decision parameters needs to be remembered when we come to discuss the relative influence of retinal location on the two sets of parameters.

In the model for single-target performance, the assumption that at the perceptual and decision levels locating and identifying are independent processes does not imply that independence will hold at the response level. In terms of the model, the probability of correct identification and location on a trial, $\left(a^{*}+a \alpha\right)\left(b^{*}+b \beta\right)$, is not equal to the product of the probability of correct identification, $\left(\mathrm{a}^{*}+\mathrm{a} \alpha\right)$ $\left(b^{*}+b\right)$, and the probability of correct location, $\left(a^{*}+a\right)\left(b^{*}+b \beta\right)$, unless the probability of a no response, $1-\left(1-a_{0}\right)\left(1-b_{0}\right)$, is identically zero $\left(a_{0}=b_{0}=0\right)$. The nonindependence at the response level raises the question of defining empirical measures of location and identity information which are independent. In an attempt to define independent measures for these two types of information, Estes et al. (1976) used as the location measure the probability of correct location contingent on correct letter identification. This measure is preferred for multiple-letter displays, and we will use it in these cases, since correctly identified letters in the response protocol can be traced to their original positions even when incorrectly located in the subject's response. This assumes, of course, that a correct target identification is unlikely to arise from a misperception of a distractor, i.e., that targets and distractors are nonconfusable. It should be noted, however, that this measure of location contingent on correct identification is not necessarily independent of the probability of correct identification, since it is possible that correctly identified targets are more likely to be correctly located than incorrectly identified targets. For this reason, we use a measure of location information based on both correctly and incorrectly identified targets for single-letter trials. We define $P(L)$ to be equal to the probability that some target (not necessarily the correct target) was placed in the target position in the subject's response. Since this measure is based on both correctly and incorrectly identified targets, we argue that it is a more complete measure of location information. Also, for the oneletter condition, the problem of tracing incorrectly identified targets in the subject's response to their original positions does not arise. This assumes, again, that targets and distractors are nonconfusable. Neither measure of location performance, of course, is applicable if there are regularities in the presented location configurations, since location responses might be based on such redundancies rather than on incoming perceptual information about target location.

We first discuss the location and identification data for trials on which a single target is presented in terms of the model proposed for this situation. Since the only measure of location performance applicable in the multiple letter conditions is location contingent on correct identification, the model cannot be extended to cover these cases. In addition, the model as stated for single-target performance does not allow for interactions which may occur between targets when multiple targets are presented. Our approach to the multiple-letter conditions involves trying to account quantitatively for the performance decrement found in identification at a given target location relative to performance at the same location in the single-letter condition. This is done by estimating the effects of such variables as the retinal location and serial position of the 
target, and the direction (peripheral or foveal) and distance of other simultaneously presented targets. A detailed description of these models is contained in the Appendix. In addition, the influence of these variables on location performance, measured as location of correctly identified targets, is considered qualitatively.

\section{METHOD}

\section{Subjects}

The 45 Stanford University students who served as subjects received credit toward introductory psychology. Subjects had normal vision and participated in one test session, which lasted approximately $50 \mathrm{~min}$. Subjects were randomly assigned to one of three conditions and were tested individually.

\section{Apparatus}

The experiment was run using a programmable CRT display (Imlac Corporation PDS-1) interacting with a PDP-10 timesharing computer system. The test display appeared on the screen in green characters against a dark gray background. The screen was viewed from a distance of approximately $50 \mathrm{~cm}$. The subjects wrote their responses on a separate response form.

\section{Stimulus Materials}

One complete set of stimulus materials was constructed for each of the three conditions. The premask and postmask array for all conditions consisted of a horizontal string of 12 noise characters (dollar signs) with a small $x$ in the center of the array. The array subtended a horizontal visual angle of $8.75^{\circ}$ and a vertical angle of $.75^{\circ}$ when viewed from a distance of $50 \mathrm{~cm}$. The noise characters and letters measured $.5^{\circ}$ wide and $.75^{\circ}$ high. The central $\times$ measured $.5^{\circ}$ wide and $.5^{\circ}$ high. Intercharacter spaces were $.25^{\circ}$, except that the $\times$ was immediately adjacent to its flanking noise characters.

One-letter condition. Trials were of two types, letter trials and blank trials. On letter trials, the stimulus array was identical to the premask and postmask arrays, except that 1 of the 10 central noise characters was replaced by an uppercase letter drawn at random from a collection of 21 letters $(A, E, I, O$, and $U$ excluded). On blank trials, the stimulus array was constructed by replacing 1 of the 10 central noise characters with a blank. Three blocks of 60 trials were prepared, each block containing 40 letter trials and 20 blank trials. In a block of trials, a letter appeared in each of the 10 possible positions four times and a blank appeared in each of the 10 possible positions twice.

Two-letter condition. Only letter trials were presented in this condition. A stimulus array was produced by replacing 2 of the 10 central noise characters by two different uppercase letters drawn at random from the collection of 21 letters. Both letters appeared in either the left half of the array or the right half of the array and were in :arious positional configurations. The positional configurations were such that over a block of trials letters appeared in each of the 10 possible letter positions exactly eight times. Three blocks of 40 trials were constructed.

Three-letter condition. The three-letter condition is similar to the two-letter condition, except that, on each trial, three letters were presented. Within a block, letters appeared in each of 10 possible letter positions exactly 12 times, and three blocks of 40 trials were constructed.

\section{Procedure}

At the start of the test session, the subjects were given instructions about their task. They were told to which of the three conditions they had been assigned, i.e., how many letters would appear on each trial, and that, on each trial, the following sequence of events would occur: (1) The word READY would appear centered on the CRT display screen. (2) The subject would then press the spacebar on the typewriter-like keyboard in front of the CRT screen. (3) The premask array would appear centered on the screen. (4) After the subject had fixated on the small $x$ in the center of the array, the subject would again press the spacebar. (5) In the one-letter condition, either one blank or one letter would immediately appear in place of one of the noise characters and would remain for $80 \mathrm{msec}$. In the two- and three-letter conditions, two or three letters replaced noise characters for $80 \mathrm{msec}$. (6) The test stimulus would then be followed by the postmask array, which remained on the screen for $500 \mathrm{msec}$. (7) Finally, the word RESPONSE would appear on the screen and the subject would write his response on the response form provided. The word RESPONSE would remain on for a period of $1 \mathrm{~min}$ or until the subject initiated the next trial by pressing the space bar again.

The subjects were instructed to indicate what letters they saw and where they saw them by writing the letters in the appropriate letter positions on the response form. The subjects in the one-letter condition were instructed to draw a small square in the appropriate position on blank trials to indicate that a blank had been presented in that position. The subjects were encouraged to guess on the basis of partial information, but to leave the position blank if they had no idea what letter had been presented there.

In a test session, 10 practice trials were followed immediately by the three blocks of trials. The blocks were randomly ordered for each subject and trials were randomly ordered within the blocks.

\section{RESULTS}

\section{Summary of Data}

A brief description of the scoring system will be given before presenting the results. Identification performance is measured as the proportion of trials on which the target presented in a given retinal location is contained somewhere in the subject's response, not necessarily in the correct location. This measure is denoted $\mathrm{P}(\mathrm{I})$. Two measures of location performance are used. The first, denoted $\mathrm{P}(\mathrm{L})$ and used in the one-letter condition only, is the proportion of trials on which any target (i.e., letter or blank target) is placed in the target position in the subject's response, not necessarily the correct target. The second measure of location performance used is the distribution of response locations for correctly identified targets. This measure is used in the two- and three-letter conditions and takes the form of a relative frequency histogram of response locations.

\section{One-Letter Condition}

Figure 1 shows $\mathrm{P}(\mathrm{I})$ and $\mathrm{P}(\mathrm{L})$ plotted as a function of retinal location (i.e., number of target positions away from the fixation point) for target letters and target blanks in the one-letter condition. The values shown are the averages of the individual subject proportions.

$\mathbf{P ( I ) . ~ A n ~ a n a l y s i s ~ o f ~ v a r i a n c e ~ w a s ~ d o n e ~ o n ~ t h e ~}$ identification scores, $\mathrm{P}(\mathrm{I})$, for target letters using individual subject proportions as scores. Retinal Location $(1,2,3,4$, or 5$)$ was found to have a significant effect, $F(4,56)=3.802, p<.01$. The 


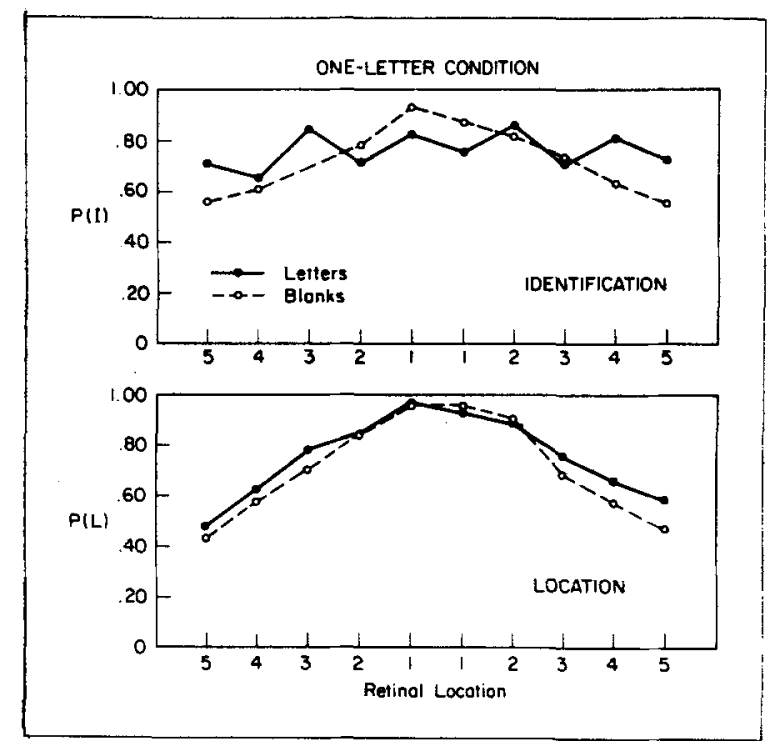

Figure 1. Identification performance, $P(I)$, and location performance, $P(L)$, for target letters and target blanks in the oneletter condition as a function of the retinal location of presentation.

main effect of Visual Field (left vs. right) was not significant, but the interaction between Visual Field and Retinal Location was significant, $F(4,56)=$ $10.870, p<.01$. The main effect of retinal location can be seen in Figure 1 as a slight decline in performance at the more peripheral retinal locations, while the interaction can be seen in Figure 1 as the saw-toothed pattern which is out of phase for the two visual fields.

An analysis of variance was also done on the identification scores, $\mathrm{P}(\mathrm{I})$, for target letters and target blanks together. The analysis resulted in no main effect of Target Type (letter vs. blank), but a significant interaction between Target Type and Retinal Location, $F(4,56)=8.763, \mathrm{p}<.01$. This effect can be seen in Figure 1 as the greater retinal location effect for blanks than for letters. A comparison with the location graphs in Figure 1 shows that the relatively large effect of retinal location on identifying blanks was similar in magnitude to the effect of retinal location on locating both letters and blanks. As before, Retinal Location and the interaction between Retinal Location and Visual Field were significant, and the effect of Visual Field was not significant. For blank trials on which some response was made, the probability that the response character was a letter was $.084, .154, .187, .236$, and .254 for Retinal Locations 1, 2, 3, 4, and 5, respectively. For letter trials on which some response was made, the probability that the response character was a blank was $.037, .026, .020, .003$, and .024 for Retinal Locations 1, 2, 3, 4, and 5, respectively.

$P(L)$. An analysis of variance was done on the location scores, $\mathrm{P}(\mathrm{L})$, for target letters and target blanks together. Target Type and Retinal Location were significant, $F(1,14)=7.553, p<.05$, and $\mathrm{F}(4,56)=26.066, \mathrm{p}<.01$, respectively; no other effects were significant. The target type effect can be seen in Figure 1 as the slightly lower location performance for blank targets than for letter targets. Again, the retinal location effect is the sharp decline in performance as the target was presented farther from the fixation point.

Independence of identification and location responses. Excluding those trials on which no response is made, a 2 by 2 table was constructed for each subject using frequencies from letter trials of the following four response outcomes: correct identification and correct location, incorrect identification and correct location, correct identification and incorrect location, and incorrect identification and incorrect location. A chi square test for independence was performed on each table separately. Only two chi square values reached significance at $\alpha=.05$, with correct letter identifications more likely to be correctly located than incorrect letter identifications. The average of the $\phi$ coefficients computed for individual subjects was .088 , corresponding to a $\chi^{2}(1)=.891, .30<\mathrm{p}<.50$, indicating that across subjects there was not a significant tendency for correct location responses to be associated with correct identification responses on those trials on which a response was made.

Location of correctly identified targets. Figure 2

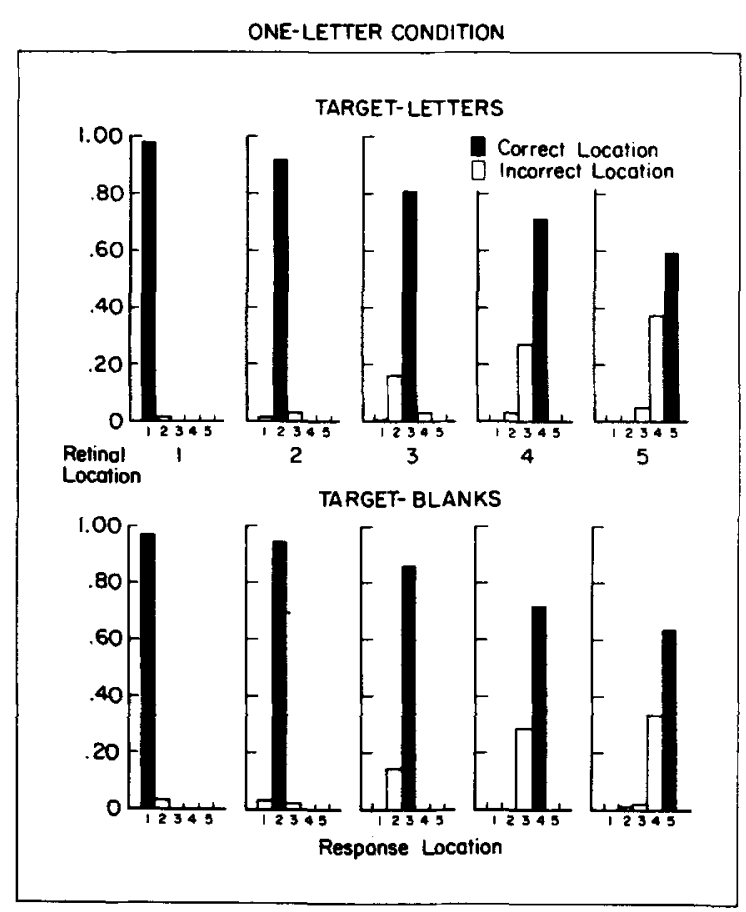

Figure 2. Location distributions for correctly identified target letters and target blanks for each retinal location of target presentation in the one-letter condition, collapsed across left and right visual fields. Shown are the probabilities of Jocating a correctly identified target in correct and incorrect response locations. 
shows the distribution of location responses for correctly identified letter and blank targets presented in each retinal location. The values shown represent the proportion of correctly identified targets located in each response position, collapsed across visual fields. In no case was a target presented in one visual field and reported in the other. The four obvious features of the data are (1) the proportion of correct location responses for correctly identified targets decreased with distance from the fovea, (2) most incorrectly located targets were placed in a foveal direction from the correct target location, (3) most incorrectly located targets were placed in neighboring retinal locations, and (4) the pattern was virtually identical for target letters and target blanks.

\section{Two- and Three-Letter Conditions}

Five trial types were used in each of the multipleletter conditions, and a sample of each trial type is shown in Table 1 . Only left visual field trials are shown; right visual field trials were mirror images.

P(I). Figure 3 presents the proportion of correct letter identifications, $P(I)$, for letter targets presented at each retinal location in the one-, two-, and three-letter conditions. For the multiple-letter conditions, the values of $P(I)$ shown are averaged across trial types. The two obvious features of the data are: (1) overall identification performance decreased as the number of letters presented on a trial increased, and (2) the effect of retinal location was larger, that is, $P(I)$ dropped off more rapidly with retinal location, when more letters were presented. In the three-letter condition, performance dropped off sharply near the fixation point and then maintained a fairly constant low level of performance. An analysis of variance done on the identification scores collapsed across trial types for the two-letter condition showed a significant Retinal Location

Table 1

Sample Trials from the Two- and Three-Letter Conditions

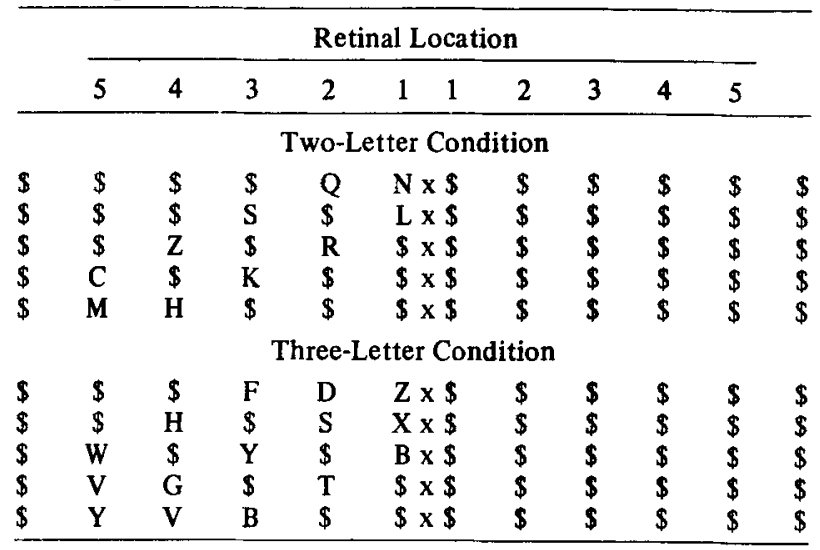

Note-Only left visual field trials are shown. Right visual field trials were mirror images.

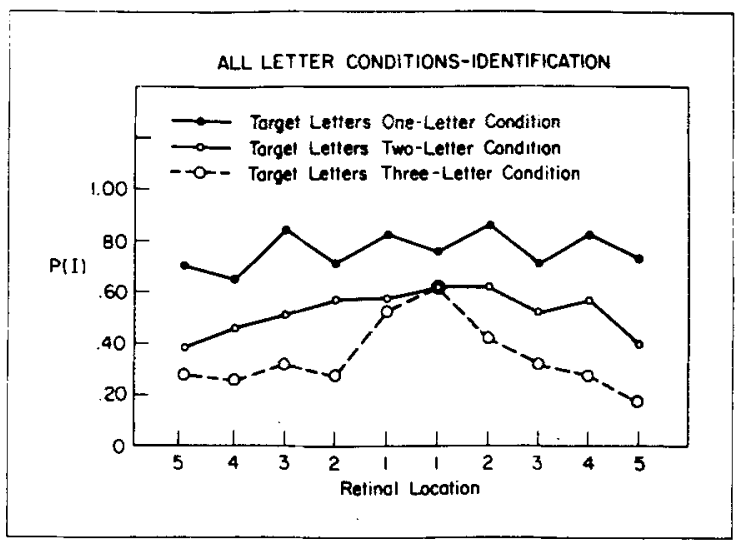

Figure 3. Identification performance, $P(I)$, for target letters in the one-, two-, and three-letter conditions as a function of the retinal location of presentation.

effect, $F(4,56)=18.297, p<.01$, and insignificant Visual Field and Retinal Location by Visual Field effects. A similar analysis for the three-letter condition showed a significant Retinal Location effect, $F(4,56)=34.088, p<.01$, and an insignificant Visual Field effect. Retinal Location by Visual Field, however, was significant, $F(4,56)=9.559, \mathrm{p}<.01$. An analysis of variance done for trial type used as scores the average performance on each trial type. For the two-letter condition, Trial Type was significant, $F(4,56)=39.646, p<.01$, Visual Field was nonsignificant, and Trial Type by Visual Field was significant, $F(4,56)=5.640, p<.01$. For the threeletter condition, Trial Type was significant, $F(4,56)=$ $55.868, \mathrm{p}<.01$, Visual Field was nonsignificant, and Trial Type by Visual Fieid was significant, $F(4,56)=$ $3.811, \mathrm{p}<.01$.

Serial position. Identification performance can be compared across trial types with respect to the serial position of the target, where serial position as used here is defined as follows. Of the letters which are presented on a trial, the letter presented closest to the fixation point is said to be in Serial Position 1 . A letter is said to be in Serial Position 2 (3) if there was one (two) letter(s) presented between it and the fixation point on the trial. Thus, serial position is defined as distance away from the fixation point and is defined only with respect to letter positions (i.e., mask character positions are ignored). Table 2 shows $P(I)$ as a function of the serial position of the target letter for the two- and three-letter conditions for each visual field. In the two-letter condition, P(I) was larger for the letter in Serial Position 2 in the left visual field, but larger for the letter in Serial Position 1 in the right visual field. A similar bias in favor of the farthest left letter is also apparent in the threeletter condition. Performance on the letter in Serial Position 3 was better in the left visual field than in the right visual field, whereas performance on the letter in Serial Position 1 was better in the right visual 
Table 2

Serial Position Data for Two- and Three-Letter Conditions

\begin{tabular}{cccc}
\hline \multirow{3}{*}{$\begin{array}{c}\text { Serial } \\
\text { Position }\end{array}$} & $\begin{array}{c}\text { Left Visual } \\
\text { Field }\end{array}$ & $\begin{array}{c}\text { Right Visual } \\
\text { Field }\end{array}$ & Average \\
\hline & \multicolumn{4}{c}{ Two-Letter Condition } \\
1 & .480 & .587 & .534 \\
2 & .519 & .499 & .509 \\
& Three-Letter Condition & \\
1 & .403 & .551 & .479 \\
2 & .228 & .306 & .267 \\
3 & .386 & .261 & .324 \\
\hline
\end{tabular}

field than in the left visual field. The serial position data for the three-letter condition, however, was dominated by a concave pattern where $\mathrm{P}(\mathrm{I})$ was largest for the most foveal letter (Serial Position 1), next largest for the most peripheral letter (Serial Position 3), and smallest for the central letter (Serial Position 2).

Interletter distance. Identification performance, $P(I)$, can also be compared across trial types with respect to the average interletter distance of the letters presented on the trial. In the two-letter condition, the interletter distance was either one or two retinal locations. The average $P(I)$ for these interletter distances was .526 and .519 , respectively, and these proportions were not significantly different from each other. In the three-letter conditions, the average interletter distances were $1,1.5$, or 2 retinal locations. The average $P(I)$ for these interletter distances was $.392, .340$, and .319 , respectively. Average performance on trials with interletter distance 1 was significantly different from average performance on trials with interletter distance $1.5, \mathrm{t}(\mathrm{df}=58)=$
$2.288, p<.05$, and average performance on trials with interletter distance 2 was significantly different from average performance on trials with interletter distances 1 and 1.5 combined, $t(d f=73)=1.961$, $\mathrm{p}<.05$.

Location of correctly identified targets. Figure 4 shows the distribution of location responses for correctly identified letters. Two types of location errors are shown, those in which the correctly identified target was placed in another target's position, and those in which the correctly identified target was placed in a nontarget position. Targets which were incorrectly located tended to (1) move foveally from their correct location, and (2) move to other target positions. As can be seen from Figure 4 , there are a number of similarities between the probability of correctly locating a correctly identified letter and the probability of correctly identifying a letter. First, the probability of correct location decreased as the distance from the fixation point increased, and also as the total number of letters on a trial increased. Second, as the average interletter distance on a trial increased, the probability of correctly locating a correctly identified target decreased. Third, the presence of a second target in a peripheral direction caused a larger decrease in performance than if the second letter was in a foveal direction. Finally, in the three-letter condition, the serial position effect for target location was similar to the serial position effect for target identification, with performance on the center letter (Serial Position 2) impaired relative to the end letters (Serial Positions 1 and 3 ).

Effect of an incorrectly located target on identification. One final question of interest is
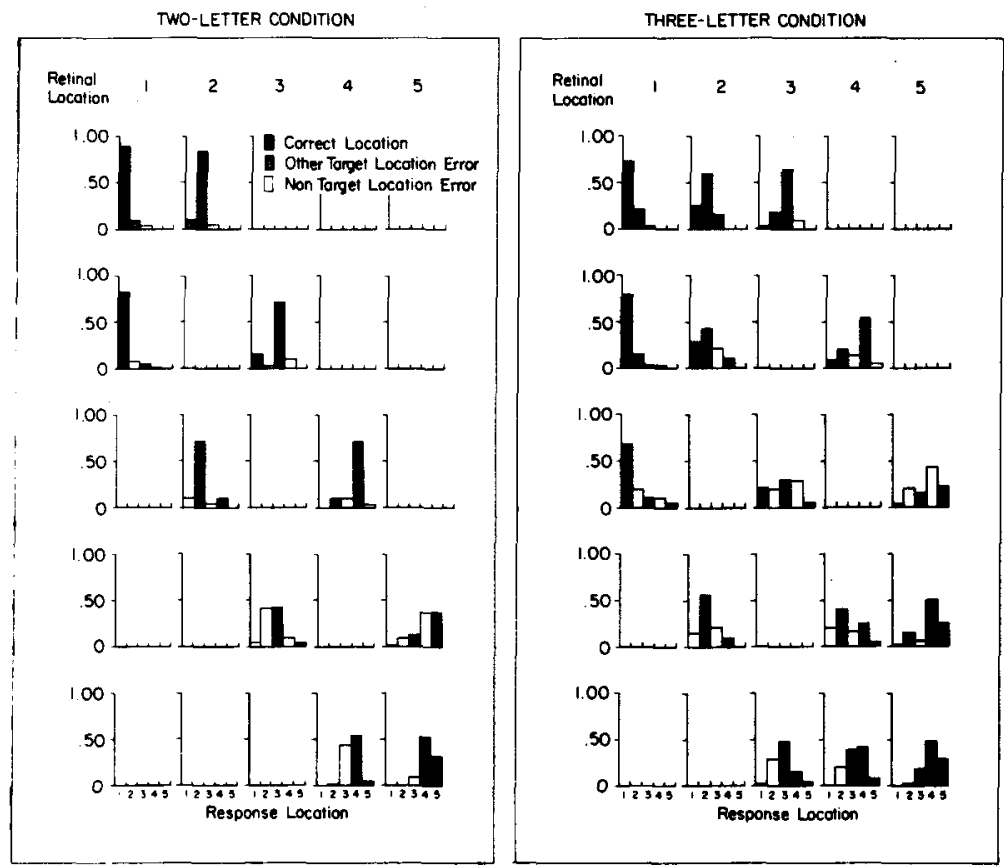

Figure 4. Location distributions for correctly identified target letters for each retinal location of target presentation in the two- and three-letter conditions, collapsed across left and right visual fields. Location distributions for the different trial types are shown on separate lines. Shown are the probabilities of locating correctly identified targets in correct response locations, incorrect response locations where some other target was presented on the trial, and incorrect response locations where no target was presented on the trial. 
whether a target moving to a second target's position was associated with a decreased probability that the second target was correctly identified on the trial. For simplicity, we consider the data from the two-letter condition only. Across subjects, the observed total number of trials on which one letter moved to the other letter's position was 249. On 76 of these trials, the second letter appeared somewhere in the subject's response (displaced from its correct location), whereas on 173 of these trials, the second letter was not correctly identified on the trial. A $\chi^{2}(1)=3.270, .05<\mathrm{p}<.10$, was computed where the predicted values were based on the relative proportion of trials on which two vs. one letter was correctly identified $(p=.36$ and $p=.64$, respectively.) There was a tendency, then, for a target not to be correctly identified if a second target was incorrectly located in its response location.

\section{Applications of Models to Data \\ One-Letter Condition}

Recall our assumptions that the quality of identification information on a trial is independent of the quality of location information, and that, with respect to each type of information, the subject is in one of three sensory states $D_{0}, D$, and $D^{*}$, corresponding to no useful information, partial or fallible information, and perfect information. The probabilities of being in these states are $a_{0}, a$, and $a^{*}$ for item information and $b_{0}, b$, and $b^{*}$ for location information. The probability of correctly identifying (locating) the target is $0, \alpha, 1(0, \beta, 1)$ for states $D_{0}, D, D^{*}$, respectively. If the subject is in state $D_{0}$ with respect to either item or location information, no response is made.

On each trial in the one-letter condition, there are five possible response outcomes: no response, incorrect identification and incorrect location, correct identification and incorrect location, incorrect
Table 3

Parameter Estimates for One-Letter Condition Model

\begin{tabular}{cccc}
\hline Type of Parameter & Parameter & \multicolumn{1}{l}{ Slope } & Intercept \\
\hline & Letters & & \\
& $\mathrm{a}^{\mathrm{a}}$ & & \\
Identification & $\mathrm{a}$ & .001 & .519 \\
& $\alpha$ & -.024 & .484 \\
Location & $\mathrm{b}^{*}$ & -.037 & .351 \\
& $\mathrm{~b}$ & .019 & .651 \\
& $\beta$ & -.124 & .985 \\
Identification & $\mathrm{a}$ & -.010 & .552 \\
& $\alpha$ & -.003 & .507 \\
& $\mathrm{a}^{*}$ & -.088 & .826 \\
Location & $\mathrm{b}^{*}$ & -.046 & .434 \\
& $\mathrm{~b}$ & .000 & .503 \\
& $\beta$ & -.134 & .972 \\
\hline
\end{tabular}

Note-Parameters are written in the form: Parameter $=$ Intercept + Slope $(j-1)$, where $j$ is retinal location $(j=1,2,3,4,5)$. $a_{\text {Loss function }-.00343}$

$\mathrm{b}_{\text {Loss function }}=.00705$

identification and correct location, and both correct identification and correct location. In terms of the underlying parameters of the model, the probability of each of these outcomes is $1-\left(1-a_{0}\right)\left(1-b_{0}\right)$, $a(1-\alpha) b(1-\beta), \quad\left(a^{*}+a \alpha\right) b(1-\beta), \quad a(1-\alpha)$ $\left(b^{*}+b \beta\right)$, and $\left(a^{*}+a \alpha\right)\left(b^{*}+b \beta\right)$, respectively. Assuming that a parameter is a linear function of distance (j) from the fixation point, each parameter can be written in the form Parameter = Intercept + Slope $(j-1), j=1,2,3,4,5$, since no letter was presented at the fixation point $(j=0)$. We obtained estimates of the slopes and intercepts for each parameter which best accounted for the relative frequencies of the five response outcomes at the five retinal locations. ${ }^{1}$ These parameter estimates were computed separately for letter trials and blank trials and are shown in Table 3 together with the value of the loss function. Table 4 shows the predicted

Table 4

Predicted and Observed Proportions of Trials in Each Response Category

\begin{tabular}{|c|c|c|c|c|c|c|c|c|c|c|}
\hline \multirow{3}{*}{$\begin{array}{l}\text { Retinal } \\
\text { Location }\end{array}$} & \multicolumn{10}{|c|}{ Response Outcome } \\
\hline & \multicolumn{2}{|c|}{ No Response } & \multicolumn{2}{|c|}{$\overline{\mathrm{I}}$ and $\overline{\mathrm{L}}$} & \multicolumn{2}{|c|}{$\mathrm{I}$ and $\mathrm{L}$} & \multicolumn{2}{|c|}{ I and I } & \multicolumn{2}{|c|}{$I$ and $L^{*}$} \\
\hline & Pred. & Obs. & Pred. & Obs. & Pred. & Obs. & Pred. & Obs. & Pred. & Obs. \\
\hline & \multicolumn{10}{|c|}{ Letter Trials } \\
\hline $\begin{array}{l}1 \\
2 \\
3 \\
4 \\
5\end{array}$ & $\begin{array}{l}.030 \\
.038 \\
.046 \\
.054 \\
.063\end{array}$ & $\begin{array}{l}.025 \\
.036 \\
.033 \\
.053 \\
.056\end{array}$ & $\begin{array}{l}.002 \\
.017 \\
.036 \\
.059 \\
.087\end{array}$ & $\begin{array}{l}.017 \\
.028 \\
.044 \\
.092 \\
.106\end{array}$ & $\begin{array}{l}.162 \\
.158 \\
.150 \\
.138 \\
.122\end{array}$ & $\begin{array}{l}.164 \\
.147 \\
.144 \\
.128 \\
.111\end{array}$ & $\begin{array}{l}.008 \\
.077 \\
.150 \\
.225 \\
.302\end{array}$ & $\begin{array}{l}.011 \\
.064 \\
.147 \\
.211 \\
.300\end{array}$ & $\begin{array}{l}.798 \\
.710 \\
.619 \\
.524 \\
.426\end{array}$ & $\begin{array}{l}.783 \\
.729 \\
.631 \\
.517 \\
.428\end{array}$ \\
\hline 5 & \multicolumn{10}{|c|}{ Blank Trials } \\
\hline $\begin{array}{l}1 \\
2 \\
3 \\
4 \\
5\end{array}$ & $\begin{array}{l}.008 \\
.068 \\
.127 \\
.185 \\
.241\end{array}$ & $\begin{array}{l}.011 \\
.061 \\
.111 \\
.178 \\
.256\end{array}$ & $\begin{array}{l}.001 \\
.011 \\
.027 \\
.048 \\
.075\end{array}$ & $\begin{array}{l}.011 \\
.028 \\
.072 \\
.061 \\
.089\end{array}$ & $\begin{array}{l}.082 \\
.107 \\
.122 \\
.127 \\
.122\end{array}$ & $\begin{array}{l}.072 \\
.117 \\
.094 \\
.133 \\
.100\end{array}$ & $\begin{array}{r}.014 \\
.076 \\
.130 \\
.176 \\
.215\end{array}$ & $\begin{array}{l}.022 \\
.044 \\
.117 \\
.183 \\
.206\end{array}$ & $\begin{array}{l}.896 \\
.739 \\
.595 \\
.465 \\
.347\end{array}$ & $\begin{array}{l}.883 \\
.750 \\
.606 \\
.444 \\
.350\end{array}$ \\
\hline
\end{tabular}

$* I, \bar{I}, L, \bar{L}$ indicate correct and incorrect identification and correct and incorrect location, respectively. 
and observed proportions in each of the five response categories at each retinal location for letter and blank targets.

The estimated slopes in Table 3 were used as the index of the retinal location effect. Regarding a slope of .01 or less as negligible, we can say that retinal location by itself does not affect the perceptual parameters $\left(a^{*}\right.$ and $\left.a\right)$ involved in identifying a letter or a blank, but does affect the decision parameter $(\alpha)$ involved in identifying both kinds of targets when there is only partial information about the target identity. The effect on the decision parameter, $\alpha$, is considerably larger for blanks (slope $=-.088$ ) than it is for letters (slope $=-.024$ ). These parameters estimates are consistent with the observation that the $P(I)$ vs. $j$ curve in Figure 1 is relatively flat for letters, but falls off more sharply for blanks.

Concerning the process of target location, it can be seen that there is a large effect of retinal location on the decision parameter for both letters and blanks (slopes $=-.124$ and -.135 , respectively). This is consistent with the observation that the proportion of correctly located targets decreased sharply as retinal location increased (Figure 1), and that the proportion of incorrectly located targets increased (Figure 2). There is also an effect, although smaller, of retinal location on the perceptual parameters for both kinds of targets. In terms of the model, the probability $b^{*}$ of having full perceptual information declines slightly with retinal location (slopes = -.037 and -.046 for letters and blanks, respectively). This effect is accompanied by a slight increase in the probability of having only partial information, $a$, for letters (slope $=.019$ ) but not for blanks (slope $=.000$ ).

The proposed model distinguishes between two sets of parameters, perceptual parameters and decision parameters. In terms of the model, the primary effect of retinal location on both identification and location of letter and blank targets was found in the decision parameters, rather than in the perceptual parameters, and the effect was much larger in the location parameters than in the identification parameters. This result depends somewhat on the choice of the simple linear relationship between the parameters and retinal location. It is possible that a model postulating somewhat different functions for the relationship between the parameters and retinal location would not necessarily reach the same conclusion about the locus of the retinal location effect. The choice of the simple linear relationship, however, allows the parameters of the model at each retinal location to be estimated from the data, since only a slope and intercept needs to be found for each of the six parameters. The present result should further be considered tentative due to the somewhat arbitrary nature, noted earlier, of the distinction made in the present model between perceptual and decision parameters.

\section{Two- and Three-Letter Conditions}

Accounting for the data from the two- and threeletter conditions, where interactions between presented targets may occur, is done only with respect to identification performance. ${ }^{2}$ The models for the multiple letter conditions attempt to account for the performance decrement at a given retinal location in the multiple-letter condition relative to performance at the same retinal location in the oneletter condition. A model here refers to a set of assumptions about how retinal location and position relative to other targets influence this decrement. A brief summary of those variables (for example, retinal location, serial position, interletter distance) which are found to influence this performance decrement will be given for each of the multipleletter conditions. A detailed description of the models which were found to best account for the data in the multiple-letter conditions is given in the Appendix.

Two separate models were constructed for the two-letter condition. The first of these attempted to determine which variables influenced average performance on a trial. It was found that performance decreased, that is, the performance decrement increased, as the average retinal location increased and as the average interletter distance increased. The second model attempted to account for the specific effects found on identification of a target when a second target was simultaneously presented. It was found that the probability of a correct identification was generally lower, and decreased more rapidly as retinal location increased, than in the one-letter condition. Further, performance on a letter was influenced by the relative position of the second letter. The performance decrement on a target was much larger if the second target was presented in a peripheral direction than if the second target was presented in a foveal direction, and the detrimental effect of a peripheral target increased as the distance between the two targets increased.

Two separate models were also constructed for the three-letter condition. As in the two-letter condition, the first model attempted to determine which variables influenced average performance on a trial. Again, it was found that average identification performance on a target is largely determined by average retinal location and average interletter distance, where the effect of an increase in each of these variables was a decrease in performance. The second model attempted to determine which variables influenced the level of performance on individual letters within a trial. The serial position of a target was found to be an important variable in determining the level of per- 
formance, with performance on the most peripheral target (Serial Position 3) generally high, performance on the most foveal target (Serial Position 1) also moderately high, and performance on the central target (Serial Position 2) relatively poor. A large effect of retinal location was found on end targets (Serial Positions 1 and 3) but not on central targets (Serial Position 2). In addition, specific effects of the direction and distance of neighboring targets were found. As in the two-letter condition, the presence of a neighboring peripheral target was found to have a detrimental effect on performance, which increased as the distance between the targets increased.

\section{DISCUSSION}

The models proposed constitute an attempt to quantify the effect of specific variables thought to be involved in the letter identification process. The model proposed for the one-letter condition was formulated to account for performance in the task in which a single target (letter or blank) was presented on a trial, and the task was to both identify and locate the target. This model distinguishes between perceptual and decision parameters, and while this distinction is to some extent arbitrary, it was chosen to reflect the distinction between the amount or quality of information and the efficiency of processing the available information. These parameters are specified for the identification and the location processes separately, and it is assumed that these processes are independent at the perceptual and decision levels. A response rule based on the outcome at the decision level for both types of information is specified with the result that, even though it is assumed that identification and location are independent processes at the perceptual and decision levels, the two types of information need no longer be independent at the response level.

In fitting the model to the experimental data, the parameters were assumed to be linear functions of retinal location. In this way the magnitude (slope) and the locus (perceptual vs. decision) of the retinal location effect could be determined. In terms of these estimated parameters, the retinal location effect for identifying letters was fairly small in the range of retinal locations investigated, and occurred primarily at the decision level. The retinal location effect for locating a letter was relatively large, and the locus of the effect was, again, primarily at the decision level, although a small effect was also found at the perceptual level. For targets which were blanks, the estimated location parameters were similar to those for letter targets. The decision parameter for identification of blank targets, however, showed a much larger retinal location effect than the decision parameter for letters.
Two aspects of our analysis argue that locating and identifying letter targets are independent processes, at least when only one letter is presented on a trial. First, a good fit to the one-letter location and identification data was achieved by assuming independence of the two types of information at the perceptual and decision levels. Secondly, a much larger retinal location effect was found on location performance than on identification performance. Additionally, the substantial superiority of identification over location performance on letter targets at peripheral retinal locations argues against the notion that identification is strongly dependent on the ability to accurately locate the target, and this is similar to the conclusion reached by Logan $(1975 a, b)$. This is not to say, however, that there may not be variables which affect the two performance measures similarly, as will be seen in the discussion of the multiple letter conditions.

The situation with regard to blank targets is, however, somewhat different and may indicate an interdependence between the identification and location processes in this case. Evidence for this comes from the observation that, for blank targets, estimates of the identification and location parameters are very similar. It might be assumed that this interdependence arises since the difficulty in both identifying and locating a blank is related to the difficulty of correctly assigning the perceived features of the neighboring noise characters to their correct locations. That is, identifying and locating a blank target are both influenced by the location uncertainty associated with noise characters. Support for this comes from the observation that on blank target trials on which a response is made, the proportion of (incorrect) letter responses increases with distance from the center of the fovea. This may be due to an increased tendency for features from the neighboring noise characters to be incorrectly associated with the target position as the target appears in more peripheral locations. The absence of features associated with a blank target makes its identification particularly vulnerable to confusion with noise characters, whereas a letter target, having distinct features, would be less affected by such confusions. Letter targets, then, would have a better probability of being correctly identified even if the perceived features were incorrectly located, thus predicting a much greater degree of independence for locating and identifying letter targets than blank targets.

The two- and three-letter condition models focused on the performance decrement found in identification when multiple letters were presented, that is, the difference between performance at a given retinal location in the one-letter condition and performance at the same retinal location in the multiple-letter conditions. In the multiple-letter-condition models, the performance decrement at a rarget location was 
written as a function of such variables as the retinal location and serial position of the target, and the distance and direction of the other simultaneously presented targets. The effects of these variables can be summarized as follows. The performance decrement increased with retinal location of the target, and the increase was greater (i.e., the slope was larger) when more letters were presented. Serial position was found to affect both the intercept and the slope of the function relating performance decrement to retinal location in the three-letter condition. Performance on the most peripheral letter was generally high (smallest estimated intercept for the performance decrement), somewhat lower for the most foveal letter, and lowest for center letters. The most peripheral and foveal letters were subject to large retinal location effects (large estimated slopes for the performance decrement), while center letters did not show a large effect of retinal location. The presence of a second peripherally presented letter had a large detrimental effect on identifying a given target, and the detrimental effect of a peripheral letter on performance was greater as the interletter distance increased. A related finding was that the average performance decrement on a trial increased as the range of letter locations increased. That is, average performance decreased as the interletter distance increased. This was true even though the average retinal location was constant for the different ranges of retinal locations, so that the effect of increasing the interletter distance could not be attributable to a change in the average retinal location.

A question of interest is to what extent these results indicate that either inhibition between neighboring letters or a limited-capacity attention mechanism is operating in the present experiment. The model proposed by Estes $(1972,1974)$ assumes that (1) the density of input channels to feature detectors decreases with distance from the fovea, and (2) input channels inhibit other input channels going to the same or other nearby feature detectors. These assumptions can be used to explain the observed decreases in performance found with distance from the fovea (retinal location effect) and the increased slopes found as more letters are presented. Inhibition between channels can also be used to explain the relatively poor performance on center letters (Serial Position 2 in the three-letter condition), since these letters are subject to inhibitory effects from both sides. However, it is difficult to see how these assumptions can account for the very small retinal location effect for center letters, since the model would in general predict that the retinal location effect should be larger for center letters than for end letters. More importantly, the results showing greater performance decrements for larger interletter distances are directly opposite what would be predicted by this sort of model, since increased interletter distances should result in decreased interference between the presented letters, thus, improving performance. In the present experiment, all nontarget retinal locations are filled with mask characters, including those retinal locations between target characters at larger interletter distances, and these mask characters may have some inhibitory effect on target identification. Since the total number of noise characters remained constant within each condition, it is difficult to see how, in the present experiment, the inhibitory effect of noise characters would be sufficient to explain the increased performance decrement found at larger interletter distances unless it were assumed that noise characters exerted more inhibition than target characters.

We now consider the possibility of explaining these results using the notion of a limited-capacity attention mechanism. The general model, proposed by Rumelhart (1970), assumes that the amount of attention available is limited and that, when it is shared between items, the result is a reduced processing efficiency on each item. Suppose this general model is extended by assuming (1) more attention is required at peripheral locations than at central locations for a certain level of performance, and (2) attention tends to be allotted preferentially to peripheral locations. The above assumptions would predict a decrease in performance with distance from the fixation point (retinal location effect) since peripheral letters require more attention, and attention is limited. When more letters are presented, attention is distributed over more letter positions. In addition to lowering overall performance, this may accentuate the retinal location effect, since peripheral locations, requiring more attention for a high level of performance, cannot be allotted the necessary amount of attention. The detrimental effect on performance found as interletter spacing increases might be explained if it were assumed that attention would be allotted to noise characters when they are included in the range of retinal locations occupied by target characters. The above assumptions also predict that a peripherally presented letter, which tends to be allotted more attention than a foveal letter, would detract from performance at a given location more than a foveally presented letter. Further, the more peripheral the letter, the more attention would be allotted to it, and the larger the detrimental effect at the more foveal location. Consequently, generally poor performance should be found for foveal letters when peripheral letters are also presented on the trial. In the three-letter condition, letters in Serial Positions 1 and 2 showed lower overall performance (larger intercept for the performance decrement) than letters in Serial Position 3, although this effect was not as strong for letters in Serial Position 1 as it was for letters in Serial Position 2. This may be due to the fact that in the 
present experiment, letters in Serial Position 1 were presented in a different range (more foveal) of retinal locations than letters in Serial Position 2. In those positions where the ranges overlap, performance on letters in Serial Position 1 was comparable to performance on letters in Serial Position 2 at the same retinal location. The relatively uniform performance on letters in Serial Position 2 across retinal locations might be explained if the center letter, within the range of letter positions used, rarely received enough attention to raise its identification performance above a certain minimal level. While the limited-capacity attention mechanism proposed here requires a number of specific assumptions about the allocation of attention, it is of some interest that such a mechanism can account for many aspects of the experimental results.

The location data for the multiple-letter conditions showed many of the same patterns as the identification data just discussed. In the multipleletter conditions, we considered location performance for correctly identified target letters only. Location performance, like identification performance, decreased as more letters were presented on the trial. Location performance was also affected by retinal location, and by the range of retinal locations, in the same way as identification performance. Location performance at a given position was lower if a peripheral, rather than a foveal, target was also presented on the trial. Finally, a very similar serial position effect was found in the three-letter condition, with performance on the center position being relatively poor. The similarity between the pattern of results for the two types of information argues that the process of extracting location information may have some elements in common with the process of extracting identification information. It is possible, for example, that the assumptions made about an attention mechanism operating in the identification of targets may apply equally well to the process of locating targets. Both identifying and locating targets, then, would be expected to be affected similarly by those variables which determine the amount of attention allotted to a given letter position. Once this attention has been allocated, it is assumed that, as in the one-letter condition model, the process of identifying and locating targets are carried on independently.

Is there any evidence that, in the multiple-letter conditions, identifying and locating targets are not independent processes, specifically, that positional uncertainty plays a role in the identification process as suggested by Estes et al. (1976) and Wolford (1975)? Two results suggest that positional uncertainty, with letters tending to move foveally, may have some effect on identification performance. First, when a target moves to another target's position in the subject's response, the second target tends not to be correctly identified, and, secondly, a peripheral target has a larger detrimental effect on a given target position than a foveal target. However, positional uncertainty is not sufficient to explain the increase in the effect of a peripheral letter as the distance between the letters increases. Since incorrectly located letters tend to be placed in neighboring response locations to the correct location, it is difficult to see how positional uncertainty could account for larger interaction effects at larger interletter distances. Moreover, although positional uncertainty may play some role in determining the level of identification performance, it can be argued that the detrimental effect caused by positional uncertainty is at the response level, rather than at the perceptual or decision level. The subject, having located two distinct targets in the same location, is faced with a response problem which is sometimes incorrectly resolved by placing the peripheral of the two letters in the more foveal position, and deleting the second (more foveal) letter entirely from the response. The observed effect of displaced targets, then, is entirely consistent with our assumption that locating and identifying letters are independent processes at the perceptual and decision levels. One last comment about the independence of the two types of information will be made. The results of the present experiment show that there is a tendency for correctly identified targets to be located in other target positions when they are incorrectly located. This observation may be taken in support of the hypothesis that locating and identifying are fairly independent processes, in that correct identification decisions and correct location decisions are not necessarily correctly associated with one another.

In summary, a number of conclusions can be drawn from the present investigation. First, the results of the present experiment can be better explained using the notion of a limited-capacity attention mechanism (Rumelhart, 1970), augmented with specific assumptions about how attention is allocated in linear strings of letters and noise characters, rather than using the notion of lateral interference (Estes, 1972, 1974). Second, the data are consistent with the assumption that the identification and location of targets are independent processes at the perceptual and decision levels, and that these processes may be similarly affected by those variables which determine the way in which attention tends to be allotted when multiple targets are presented. Third, in terms of the model proposed for identification and location performance when a single target is presented, the effect of retinal location on both identification and location of targets is different at the perceptual level than it is at the decision level. Fourth, the performance decrement found in identifying a target at a given retinal location when multiple letters are presented can 
be accounted for in terms of the retinal location and serial position of the target and the direction and distance of other simultaneously presented targets.

\section{APPENDIX}

The models for trials on which multiple letters are presented attempt to account for identification performance by accounting for the performance decrement at retinal location $\mathrm{j}$ in the multiple letter condition relative to performance at the same retinal location in the one-letter condition. The models presented are those models which best account for the performance decrement found when multiple letters are presented. This is done in terms of such variables as retinal location, serial position, and direction and distance of other simultaneously presented targets.

For the two-letter conditions, let us define $\mathrm{P}_{\mathrm{jk}}$ to be the observed proportion of trials on which letters were presented in retinal locations $\mathrm{j}$ and $\mathrm{k}$ that the letter presented in location $j$ was correctly identified. Let $\mathbf{P}_{j}$ be the expected identification performance at location $j$ when only one letter was presented. In terms of the model for the one-letter condition, the expected performance is $\mathbf{P}_{\mathbf{j}}=$ $\left(a^{*}+a \alpha\right)\left(b^{*}+b\right)$, where the parameters are computed for retinal location $\mathbf{j}$. Let $d_{j k}=P_{j}-P_{j k}$ be the performance decrement at location $\mathrm{j}$ caused by the addition of the second letter at location $k$.

In order to determine which variables affected average identification performance on a trial, we computed for each of the five trial types the average performance $\left(P_{j k}+P_{k j}\right) / 2$, where $j$ and $k$ were the target positions on that trial. The average performance decrement is, then, $\left(P_{j}+P_{k}\right) / 2-\left(P_{j k}+P_{k j}\right) / 2$, denoted $d_{j k}\left(=\bar{d}_{k j}\right) . A$ good fit to the five data points (one for each trial type) was achieved using:

$$
\mathrm{d}_{\mathrm{jk}}=\mathrm{a} \cdot(\mathrm{j}+\mathrm{k}) / 2+\mathrm{b} \cdot[\max (\mathrm{j}, \mathrm{k})-\min (\mathrm{j}, \mathrm{k})] .
$$

The estimated parameters and the value of the loss function are given in Table 5. As can be seen from the parameter estimates, average identification performance decreases, that is, the performance decrement increases, as average retinal location increases $(a=.06366)$ and as the range of letter locations (interletter distance) increases $(b=.03332)$.

In order to quantify the specific effects of a second target in location $\mathbf{k}$ on identification of a target in location $\mathrm{j}$, a second, interaction model was formulated. Let us define $w=|j-k|$, the separation of the two target letters. Also, let us define a variable $r$ where $r=1$ if $j<k$ and $r=2$ if $k<j$. This variable, $r$, indicates the direction (whether peripheral or foveal) of the target at $k$ from the target at $\mathrm{j}$. A fairly good fit to the 10 data points (two letter positions for each of the five trial types) was achieved using:

$$
d_{j k}=h_{0}+h_{1} \cdot j+h(r) \cdot w .
$$

The estimated parameters and the value of the loss function are shown in Table 5. The intercept value $\left(\mathrm{h}_{0}=.09029\right)$ can be interpreted as the overall effect of having two letters rather than one. This value indicates that per- formance is lower in general in the two-letter condition than in the one-letter condition. The estimated value of the slope $\left(h_{1}=.04802\right)$ indicates that the difference between the two conditions increased as retinal location increased. Further, there were specific effects due to the presence of the second letter. If the second letter was presented peripherally, it tended to depress performance $[\mathrm{h}(\mathrm{l})=.03487]$ and the detrimental effect was larger for larger interletter distances. However, if the second letter was presented foveally, relative performance was slightly better $[j(2)=$ $-.01771]$, and this improvement tended to increase as interletter distance increased.

For the three-letter condition, let us define $\mathbf{P}_{\mathbf{j k m}}$ to be the observed proportion of trials on which letters were presented in retinal locations $j, k$, and $m$ that the letter presented in location $\mathrm{j}$ was correctly identified. As before, let $P_{j}$ be the expected identification performance at location $j$ in the one-letter condition and $d_{j k m}=P_{j}-P_{j k m}$ be the performance decrement at position $\mathrm{j}$.

As in the two-letter condition, we first try to account for average performance on a trial. The average performance on a trial was computed as $\left(\mathbf{P}_{\mathbf{j k m}}+\mathbf{P}_{\mathbf{k j m}}+\mathbf{P}_{\mathbf{m j k}}\right) / 3$, and the average performance decrement at $\left(\mathbf{P}_{\mathbf{j}}+\mathbf{P}_{\mathbf{k}}+\mathbf{P}_{\mathbf{m}}\right) /$ $3-\left(P_{j k m}+P_{k j m}+P_{m k j}\right) / 3$, denoted $d_{j k m}$. As before, the model which involved average retinal location and range of retinal locations provided a good fit to the five data points (average performance for the five different trial types). In Table 6, we present the estimated parameters and the value of the loss function for this model given by:

$$
\begin{aligned}
\bar{d}_{j k m}= & a \cdot(j+k+m) / 3+b \\
& \cdot[\max (j, k, m)-\min (j, k, m)] .
\end{aligned}
$$

Average retinal location has an effect $(a=.10519)$, and this effect is larger than in the two-letter condition. Also, the range of the presented letter locations has an effect $(\mathrm{b}=.03551)$ which is similar in magnitude to the twoletter condition.

Finally, we were interested in quantifying the effect of the serial position of the target at $j$, and the specific interaction effects of the other simultaneously presented targets on the target in location $\mathrm{j}$. Let $\mathrm{c}=1$, if $\mathrm{j}<\mathrm{k}$ and $\mathrm{j}<\mathrm{m}$, let $\mathrm{c}=2$, if $\mathrm{k}<\mathrm{j}<\mathrm{m}$ or $\mathrm{m}<\mathrm{j}<\mathrm{k}$, and $\mathrm{c}=3$, if $k<j$ and $m<j$. In addition, let $r_{j k}=1$, if $j<k$, and

\begin{tabular}{|c|c|}
\hline Parameter & Parameter Estimate \\
\hline \multicolumn{2}{|c|}{ Average Performance Modela } \\
\hline \multicolumn{2}{|c|}{$\bar{d}_{j k}=a \cdot(j+k) / 2+h \cdot[\max (j, k)-\min (j, k)]$} \\
\hline $\begin{array}{l}\mathbf{a} \\
\mathbf{b}\end{array}$ & $\begin{array}{l}.06366 \\
.03332\end{array}$ \\
\hline \multicolumn{2}{|c|}{ Interaction Model ${ }^{\mathbf{b}}$} \\
\hline \multicolumn{2}{|c|}{$d_{j k}=h_{0}+h_{1} \cdot j+h(r) \cdot w$} \\
\hline $\begin{array}{l}h_{0} \\
h_{\mathrm{s}} \\
h(1) \\
h(2)\end{array}$ & $\begin{array}{r}.09029 \\
.04802 \\
.03487 \\
-.01771\end{array}$ \\
\hline
\end{tabular}
$r_{j k}=2$, if $k<j$, indicating the direction (peripheral or

Table 5

Parameter Estimates for Two-Letter Condition Models

${ }^{\mathrm{a}}$ Loss Function $=.01633$

$\mathrm{b}_{\text {Loss Function }}=.04817$ 
Table 6

Parameter Estimates for Three-Letter Condition Models

\begin{tabular}{|c|c|}
\hline Parameter & Parameter Estimate \\
\hline \multicolumn{2}{|c|}{ Average Performance Model ${ }^{\mathbf{a}}$} \\
\hline \multicolumn{2}{|c|}{$\bar{d}_{j k m}=a \cdot(j+k+m) / 3+b \cdot[\max (j, k, m)-\min (j, k, m)]$} \\
\hline $\begin{array}{l}\mathrm{a} \\
\mathrm{b}\end{array}$ & $\begin{array}{l}.10519 \\
.03551\end{array}$ \\
\hline \multicolumn{2}{|c|}{ Interaction Model $^{b}$} \\
\hline$d_{j k m}=h_{0}(c)+h_{1}(c$ & $\cdot w_{j k}+h\left(r_{j m}, n_{j m}\right) \cdot w_{j m}$ \\
\hline $\begin{array}{l}\mathrm{h}_{0}(1) \\
\mathrm{h}_{0}(2) \\
\mathrm{h}_{0}(3) \\
\mathrm{h}_{1}(1) \\
\mathrm{h}_{1}(2) \\
\mathrm{h}_{1}(3) \\
\mathrm{h}(1,1) \\
\mathrm{h}(1,2) \\
\mathrm{h}(2,1) \\
\mathrm{h}(2,2)\end{array}$ & $\begin{array}{r}-.02688 \\
.16396 \\
-.19747 \\
.15552 \\
.05720 \\
.12356 \\
.07865 \\
-.00524 \\
.04163 \\
.00520\end{array}$ \\
\hline
\end{tabular}

${ }^{\mathrm{a}}$ Loss function $=.00042$

${ }^{b}$ Loss function $=.01419$

foveal) of the target at $k$ from the target at $j$. Also define $n_{j k}=1$, if the third letter in position $m$ is not between the letters in positions $\mathbf{j}$ and $\mathbf{k}$, i.e., if the targets at $\mathbf{j}$ and $k$ are neighboring targets, and let $n_{j k}=2$, if the third letter is between the targets in positions $\mathrm{j}$ and $\mathrm{k}$, i.e., the target at $\mathrm{m}$ is an "intervening" target. Let $w_{j k}=|j-k|$ be the intertarget distance. The variables $r_{j m}, n_{j m}$, and $w_{j m}$ are defined similarly. A good fit was found for the 15 data points (three letter locations for the five trial types) using the model:

$$
\begin{aligned}
d_{j k m}= & h_{0}(c) \\
& +h_{1}(c) \cdot j+h\left(r_{j k}, n_{j k}\right) \cdot w_{j k}+h\left(r_{j m}, n_{j m}\right) \cdot w_{j m} .
\end{aligned}
$$

The estimated parameters and the values of the loss function are given in Table 6. Performance on Serial Position 3 targets was relatively high, $\left[\mathrm{h}_{0}(3)=-.19747\right]$, on Serial Position 1 targets, moderately high $\left[h_{0}(1)=-.02688\right]$, and on Serial Position 2 targets, relatively poor $\left[\mathrm{h}_{0}(2)=\right.$ .16396]. Retinal location had a larger effect on end letters $\left[h_{1}(1)=.15552\right.$ and $\left.h_{1}(3)=.12356\right]$ and a relatively small effect on center letters $\left[\mathrm{h}_{1}(2)=.05720\right]$. In addition, the interaction parameters show a large detrimental effect of a neighboring peripheral letter $[\mathrm{h}(1,1)=.07865]$ and $\mathrm{a}$ moderate effect of a neighboring foveal letter $[\mathrm{h}(2,1)=$ .04163]; both effects increase as the distance between the targets increases. Nonneighboring targets have little effect $[\mathrm{h}(1,2)=-.00524$ and $\mathrm{h}(2,2)=.00520]$.

\section{REFERENCES}

Breitmeyer, B., \& GANZ, L. Implications of sustained and transient channels for theories of visual pattern masking, saccadic suppression, and information processing. Psychological Review, 1976, 83, 1-37.

Collins, J. F., \& Eriksen, C. W. The perception of multiple simultaneously presented forms as a function of foveal spacing.
Perception \& Psychophysics, 1967, 2, 369-373.

Coltheart, M. Visual information processing. In P. C. Dodwell (Ed.). New horizons in psychology 2. Harmondsworth: Penguin, 1972.

Crovitz, H. F., \& Schiffman, H. R. Visual field and the letter span. Journal of Experimental Psychology, 1965, 70, 218-223.

Eriksen, B. A., \& ERIKSEN, C. W. Effects of noise letters upon the identification of a target letter in a nonsearch task. Perception \& Psychophysics, 1974, 16, 143-149.

Estes, W. K. Interactions of signal and background variables in visual processing. Perception \& Psychophysics, 1972, 12, 278-286.

Estes. W. K. Redundancy of noise elements and signals in visual detection of letters. Perception \& Psychophysics, 1974, 16, 53-60.

Estes, W. K., Allmeyer, D. H., \& Reder, S. M. Serial position functions for letter identification at brief and extended exposure durations. Perception \& Psychophysics, 1976, 19, 1-15.

Estes, W. K., \& Wolford, G. L. Effects of spacing on report from tachistoscopically presented letter strings. Psychonomic Science, 1971, 25, 77-80.

GARDNER, G. T. Evidence for independent parailel channels in tachistoscopic perception. Cognitive Psychology, 1973, 4, 130-155.

Krantz, D. H. Threshold theories of signal detection. Psychological Review, 1969, 76, 308-324.

LoGAN, G. D. On the independence of naming and locating masked targets in visual search. Canadian Journal of Psychology, 1975, 29, 51-58. (a)

LogAN, G. D. On the relation between identifying and locating masked targets in visual search. Quarterly Journal of Experimental Psychology, 1975, 27, 451-457. (b)

LUCE, R. D. A threshold theory for simple detection experiments. Psychological Review, 1963, 70, 61-79.

Merikie, P. M., Coltheart, M., \& Lowe, D. G. On the selective effects of a patterned masking stimulus. Canadian Journal of Psychology, 1971, 25, 264-79.

Merikle, P. M., \& Coltheart, M. Selective forward masking. Canadian Journal of Psychology, 1972, 26, 296-302.

Rumelhart, D. E. A multicomponent theory of the perception of briefly exposed visual displays. Journal of Mathematical Psychology, 1970, 7, 191-218.

SHAw, P. Processing of tachistoscopic displays with controlled order of characters and spaces. Perception \& Psychophysics, $1969,6,257-266$.

Shiffrin, R. M., \& Geisler, W. S. Visual recognition in a theory of information processing. In R. L. Solso (Ed.), Contemporary issues in cognitive psychology: The Loyola symposium. Washington, D.C: Winston, 1973.

SPERLING, G. The information in brief visual presentations. Psychological Monographs, 1960, 74(No. 498), 1-29.

TAYLOR, S. G., \& BROWN, D. R. Lateral visual masking: Supraretinal effects when viewing linear arrays with unlimited viewing time. Perception \& Psychophysics, 1972, 12, 97-99.

TOWNSEND, J. T. Theoretical analysis of an alphabetic confusion matrix. Perception \& Psychophysics, 1971, 9, 40-50.

Townsend, J. T., TAYloR, S. G., \& BRown, D. R. Lateral masking for letters with unlimited viewing time. Perception \& Psychophysics, 1971, 10, 375-378.

WhITE, M. J. Retinal locus and the letter-span error function. Perception \& Psychophysics, 1970, 8, 107-109.

WOLFORD, G. Perturbation model for letter identification. Psychological Review, 1975, 82, 184-199.

Wolford, G., \& Hollingsworth, S. Evidence that short-term memory is not the limiting factor in the tachistoscopic full report procedure. Memory \& Cognition, 1974, 2, 796-800. (a)

WOLFORD, G., \& HoLlingsworTH, S. Lateral masking in visual information processing. Perception \& Psychophysics, 1974, 16. 315-320. (b)

Wolford, G., \& Hollingsworth, S. Retinal location and string position as important variables in visual information processing. Perception \& Psychophysics, 1974, 16, 437-442. (c) 


\section{NOTES}

1. The parameters were estimated using a computer program GRIDS, which searches the parameter space for values which minimize the specified loss function. The loss function used in all cases was the sum of squared deviations between the predicted and observed values.

2. The three-state model cannot easily be extended to apply to data in the multiple-letter condition. One reason for this is that the measure of location information used in the single-letter condition cannot be employed in the multiple-letter condition. This is true since, in the multiple-letter conditions, when an incorrectly identified target appears in a target location in the subject's response, it is impossible to know whether it results from an incorrect identification of the target in that location or from the perception of some other target presented on the trial which is incorrectly located in the subject's response. A second reason that the model cannot easily be extended to the multiple-letter conditions is that the six parameters of the model needed at each retinal location would likely be affected by such variables as serial position and direction and distance of other simultaneously presented targets, in addition to retinal location, so that all the necessary parameters cannot be estimated from the available data.

(Received for publication April 9, 1976; revision accepted July 21,1976 .) 